\title{
Universality versus nonuniversality in asymmetric fluid criticality
}

\author{
M.A. Anisimov \\ Institute for Physical Science and Technology, University of Maryland, College Park, MD 20742, USA \\ Received January 14, 2013, in final form March 27, 2013
}

Critical phenomena in real fluids demonstrate a combination of universal features caused by the divergence of long-range fluctuations of density and nonuniversal (system-dependent) features associated with specific intermolecular interactions. Asymptotically, all fluids belong to the Ising-model class of universality. The asymptotic power laws for the thermodynamic properties are described by two independent universal critical exponents and by two independent nonuniversal critical amplitudes; other critical amplitudes can be obtained by universal relations. The nonuniversal critical parameters (critical temperature, pressure, and density) can be absorbed in the property units. Nonasymptotic critical behavior of fluids can be divided into two parts, symmetric ("Isinglike") and asymmetric ("fluid-like"). The symmetric nonasymptotic behavior contains a new universal exponent (Wegner exponent) and the system-dependent crossover scale (Ginzburg number) associated with the range of intermolecular interactions, while the asymmetric features are generally described by an additional universal exponent and by three nonasymptotic amplitudes associated with mixing of the physical fields into the scaling fields.

Key words: fluids, critical point, universality, complete scaling

PACS: $64.60 . \mathrm{F}-$

\section{Introduction}

Universality of critical phenomena is one of the most fascinating concepts in physics of condensed matter [1,2] Phase transitions of strikingly different nature, such as para-ferro-magnetism, vaporization, or fluid demixing may be described by the same equation of state near the critical points if a proper ("isomorphic") set of thermodynamic variables is chosen. There are several classes of universality defined through the dimension of the order parameter. The order parameter may be a scalar, $n$-component vector or a tensor. For example, the order parameter in fluids is associated with the density or concentration (a scalar) while the order parameter in anisotropic magnetics (magnetization) is a one-component vector. The Ising model of anisotropic ferromagnets is mathematically equivalent to the lattice-gas model which describes the condensation of fluids. In the both cases, the order parameter is one-dimensional ( $n=$ 1). The isomorphism between the members of a universality class can be established by mapping the thermodynamic variables of one system onto another. In addition, the order parameter can be conserved (such as density) or non-conserved (such as magnetization). This particular nature of the order parameter affects the phase-transition dynamics.

It is well established, primarily through experiments [3, 4], that all fluids and fluid mixtures belong to the Ising-model class of universality in statics and to the conserved-order-parameter class of universality in dynamics. This universality is associated with the universal nature of critical fluctuations. The fluctuations of the order parameter diverge at the critical point. The correlation length of the order-parameter fluctuations becomes much larger than the range of intermolecular interactions, thus making the details of the intermolecular potential unimportant. Landau and Lifshitz stated in an earlier edition of "Statistical Physics" [5], "Unlike solids and gases, liquids do not allow a general calculation of their thermodynamics quantities or even their temperature dependence. The reason for this is the presence of strong interactions between the molecules of the liquid without having, at the same time, the smallness of vibrations which 
makes the thermal motions of solids so simple." Undoubtedly, this statement is not applicable to liquids in the vicinity of their critical points. Thermodynamic properties in the critical region of very different substances, such as helium isotopes and other inert gases, organic liquids and water can be theoretically predicted; they are all described by the universal power laws, also known as scaling laws, which are characterized by the universal critical exponents.

However, there are still non-universal features in the critical behavior of fluids. The critical parameters (temperature, pressure, and density) are obviously non-universal, being determined by specific intermolecular potentials. The critical temperature ranges from a few kelvins for helium isotopes to thousands of kelvins for liquid metals. This particular non-universal feature can be eliminated by reducing the properties of a particular substance by its critical parameters. Another non-universal feature is the size of the asymptotic critical region in which the universal scaling laws are valid. This size is controlled by the so-called Ginzburg number which depends on the range of intermolecular interactions. Finally, real fluids, unlike the lattice-gas/Ising model, are asymmetric with respect to the critical isochor. The fluid asymmetry causes additional specific non-asymptotic corrections to the universal critical behavior. In this paper, I present a brief overview of universal and nonuniversal contributions to the equation of sate of near-critical fluids.

\section{Universal asymptotic criticality}

The fluctuation-induced non-analytic critical behavior can be asymptotically described by scaling theory in terms of two independent scaling fields, namely, $h_{1}$ ("ordering” field) and $h_{2}$ ("thermal" field) and two conjugate scaling densities, namely, the order parameter $\phi_{1}$ (strongly fluctuating) and $\phi_{2}$ (weakly fluctuating). The third field, $h_{3}$, is the critical part of an appropriate field-dependent thermodynamic potential, which is defined as a function exhibiting a minimum at equilibrium with respect to a variation of the order parameter. The differential of the third field is

$$
\mathrm{d} h_{3}=\phi_{1} \mathrm{~d} h_{1}+\phi_{2} \mathrm{~d} h_{2} .
$$

In the scaling theory, the field potential $h_{3}$ is a homogeneous function of $h_{1}$ and $h_{2}$. Asymptotically,

$$
h_{3} \approx\left|h_{2}\right|^{2-\alpha} f^{ \pm}\left(\frac{h_{1}}{\left|h_{2}\right|^{2-\alpha-\beta}}\right),
$$

where $f^{ \pm}$is a scaling function and the superscript \pm refers to $h_{2}>0$ and $h_{2}<0$, respectively. Here and below, $\approx$ means asymptotically equal, while $\simeq$ means approximately equal. The critical point is defined by the condition $h_{1}=h_{2}=h_{3}=0$. The form of the scaling function is universal; however, it contains two thermodynamically independent (but system-specific) amplitudes. All other asymptotic amplitudes are related to the selected ones by universal relations. The critical exponents $\alpha$ and $\beta$ are universal within a class of critical-point universality. All fluids and fluid mixtures belong to the Ising-model universality class. The Ising values for $\alpha \simeq 0.109$ and $\beta \simeq 0.326$, are well established theoretically and confirmed experimentally [3, 4, 6-16]. Two Ising amplitudes, $\hat{A}_{0}$ and $\hat{B}_{0}$ are determined by the asymptotic powerlaw behavior of the two scaling densities in zero ordering field $\left(h_{1}=0\right)$ :

$$
\begin{aligned}
\phi_{1} & =\left(\frac{\partial h_{3}}{\partial h_{1}}\right)_{h_{2}} \approx \pm \hat{B}_{0}\left|h_{2}\right|^{\beta}\left(h_{2}<0\right), \\
\phi_{2} & =\left(\frac{\partial h_{3}}{\partial h_{2}}\right)_{h_{1}} \approx \frac{\hat{A}_{0}^{ \pm}}{1-\alpha} h_{2}\left|h_{2}\right|^{-\alpha}+B_{\mathrm{cr}}\left|h_{2}\right|,
\end{aligned}
$$

and of the three scaling susceptibilities, "strong” $\chi_{1}$, "weak" $\chi_{2}$, and "cross" $\chi_{12}$ in zero ordering field:

$$
\begin{aligned}
\chi_{1} & =\left(\frac{\partial \phi_{1}}{\partial h_{1}}\right)_{h_{2}} \approx \hat{\Gamma}_{0}^{ \pm}\left|h_{2}\right|^{-\gamma}, \\
\chi_{2} & =\left(\frac{\partial \phi_{2}}{\partial h_{2}}\right)_{h_{1}} \approx \hat{A}_{0}^{ \pm}\left|h_{2}\right|^{-\alpha}, \\
\chi_{12} & =\left(\frac{\partial \phi_{1}}{\partial h_{2}}\right)_{h_{1}} \approx \beta \hat{B}_{0} \frac{\left|h_{2}\right|^{\beta}}{h_{2}} \quad\left(h_{2}<0\right),
\end{aligned}
$$


where the strong susceptibility critical exponent

$$
\gamma=2-\alpha-2 \beta \simeq 1.239,
$$

and the strong susceptibility critical amplitude $\hat{\Gamma}_{0}^{ \pm}$is related to $\hat{B}_{0}$ and $\hat{A}_{0}^{ \pm}$through universal ratios as [12]:

$$
\begin{aligned}
\alpha \hat{\Gamma}_{0}^{+} \hat{A}_{0}^{+} / \hat{B}_{0}^{2} & \simeq 0.0581, \\
\hat{\Gamma}_{0}^{+} / \hat{\Gamma}_{0}^{-} & \simeq 4.8 \\
\hat{A}_{0}^{+} / \hat{A}_{0}^{-} & \simeq 0.523 .
\end{aligned}
$$

While the superscript \pm refers to the states at $h_{2}>0$ and $h_{2}<0$, the prefactor \pm in equation (3) refers to the two branches of the order parameter corresponding to $h_{1}>0$ and $h_{1}<0$ sides (in the limit $h_{1}=0$ ), respectively. The field-dependent potential $h_{3}$ is symmetric with respect to the sign of the ordering field $h_{1}$ and, hence, to the sign of the order parameter $\phi_{1}$. In these expressions $\hat{A}_{0}^{ \pm}, \hat{\Gamma}_{0}^{ \pm}$, and $\hat{B}_{0}$ are non-universal critical amplitudes. The term in $\phi_{2}$ proportional to $B_{\mathrm{cr}}$ is an analytic fluctuation-induced contribution to the second scaling density [17]. Strictly speaking, this term is not asymptotic since the term $h_{2}\left|h_{2}\right|^{-\alpha}$ dominates.

Additional universal relations connect the critical exponent $v \simeq 0.63$ of the correlation length (diverging in zero ordering field as $\xi=\xi_{0}^{ \pm}\left|h_{2}\right|^{-v}$ ) and $\alpha$,

$$
2-\alpha=d v
$$

(where $d$ is the number of space dimensions), and the amplitudes $\hat{A}_{0}^{+}$and $\xi_{0}^{+}$,

$$
A_{0}^{+} \rho_{\mathrm{c}}\left(\xi_{0}^{+}\right)^{3} \simeq 0.172
$$

This relation is known as the two-scale factor of universality [1, 12]. The ratio $\xi_{0}^{+} / \xi_{0}^{-} \simeq 1.96$ is also universal [12].

In the mean-field approximation, with $\alpha=0$ and $\beta=1 / 2$, equation (2) reduces to the asymptotic Landau expansion [18],

$$
-h_{3} \approx \frac{1}{2} a_{0} h_{2} \phi_{1}^{2}+\frac{1}{24} u_{0} \phi_{1}^{4}-h_{1} \phi_{1},
$$

where $a_{0}$ and $u_{0}$ are mean-field system-dependent amplitudes. The amplitude $a_{0}$ is unimportant. It can be eliminated by rescaling the fields $h_{3}$ and $h_{1}$ and the coupling constant $u_{0} \rightarrow u=u_{0} / a_{0}$.

In the lattice-gas model, the ordering field $h_{1}$ is associated with the chemical potential $\mu$, the thermal field $h_{2}$ is associated with the temperature $T$, and the order parameter is associated with the molecular density $\rho$, while $h_{3}$ is associated with the pressure $P$. In both, the scaling regime and the mean-field approximation, the thermodynamic properties of the lattice gas are symmetric with respect to the sign of the order parameter. Similar to the lattice-gas model, in real one-component fluids, the thermodynamic fields are the temperature $T$, the chemical potential $\mu$, and the pressure $P$, while the conjugate densities are the number density $\rho$ and the entropy density $s=\rho S$ ( $S$ is the entropy per molecule). The physical variables are interrelated by the Gibbs-Duhem relation

$$
\mathrm{d} P=\rho \mathrm{d} \mu+s \mathrm{~d} T \text {. }
$$

Consequently, the densities, namely, the molecular density and the entropy density are derived from the pressure as

$$
\rho=\left(\frac{\partial P}{\partial \mu}\right)_{T}, \quad s=\left(\frac{\partial P}{\partial T}\right)_{\mu} .
$$

In addition to the reduced density $\Delta \hat{\rho}$ and reduced temperature $\Delta \hat{T}$,

$$
\Delta \hat{\rho}=\frac{\rho-\rho_{\mathrm{c}}}{\rho_{\mathrm{c}}}, \quad \Delta \hat{T}=\frac{T-T_{\mathrm{c}}}{T_{\mathrm{c}}},
$$

it is convenient to define

$$
\Delta \hat{s}=\frac{s-s_{\mathrm{c}}}{\rho_{\mathrm{c}} k_{\mathrm{B}}}, \quad \Delta \hat{P}=\frac{P-P_{\mathrm{c}}}{\rho_{\mathrm{c}} k_{\mathrm{B}} T_{\mathrm{c}}}, \quad \Delta \hat{\mu}=\frac{\mu-\mu_{\mathrm{c}}}{k_{\mathrm{B}} T_{\mathrm{c}}},
$$


where $k_{\mathrm{B}}$ is Boltzmann's constant. In equations (17)-18) and below, the subscript "c" denotes the properties at the critical point.

As shown by Anisimov et al. [19], since in classical thermodynamics, the absolute value of entropy is arbitrary, the critical value of entropy can be chosen upon practical convenience. It is clearly seen from the basic thermodynamic relation

$$
\mathrm{d} P=\rho \mathrm{d} \mu+\rho S \mathrm{~d} T
$$

that

$$
\frac{\mathrm{d} \hat{\mu}}{\mathrm{d} \hat{T}}+\hat{S}_{\mathrm{c}}-\left(\frac{\partial \hat{P}}{\partial \hat{T}}\right)_{\mathrm{c}}=0
$$

Thus, with adopting $\hat{S}_{\mathrm{c}}=(\partial \hat{P} / \partial \hat{T})_{h_{1}=0, \mathrm{c}}$, we obtain $(\partial \hat{\mu} / \partial \hat{T})_{h_{1}=0, \mathrm{c}}=0$ meaning that in the linear approximation, the chemical potential along the vapor-liquid coexistence does not depend on temperature. With this choice of the critical entropy, we find for the critical part of pressure (the density of the grand thermodynamic potential $\Omega=-P V$ ) after subtracting its regular part

$$
\Delta \widetilde{P}=\Delta \hat{P}-\Delta \hat{\mu}-\hat{s}_{\mathrm{c}} \Delta \hat{T} .
$$

Then, asymptotically, for one-component fluids, the scaling fields have the following simple relations to the physical fields:

$$
\begin{aligned}
& h_{1}=\Delta \hat{\mu}, \\
& h_{2}=\Delta \hat{T}, \\
& h_{3}=\Delta \widetilde{P} .
\end{aligned}
$$

One can conclude that in the asymptotic regime, in addition to the system-dependent critical parameters, which can be eliminated by rescaling the units of thermodynamic properties, there are only two independent critical amplitudes. The independent critical amplitudes correspond to the relevant nonuniversal coefficients, $u$ and $g$, in the asymptotic Landau-Ginzburg Hamiltonian, given in terms of the spatially dependent order parameter $\phi_{1}(\mathbf{x})$ as [18]

$$
\not{H}=\frac{1}{2}(\Delta \hat{T}) \phi_{1}^{2}+\frac{u}{4 !} \phi_{1}^{4}+\frac{1}{2} g \phi_{1}\left(\nabla \phi_{1}\right)^{2}-h_{1} \phi_{1} .
$$

The universal scaling function $f^{ \pm}$in equation (2) for practical applications is commonly calculated from a parametric equation of state, such as the linear model [16, 20], which has been shown to be accurate to an order of $\epsilon^{2}$ in the $\epsilon$-expansion, where $\epsilon=4-d$ [21].

\section{Size of the critical region and symmetric corrections to asymptotic scaling laws}

The universal scaling laws discussed in the previous Section are valid only asymptotically, very close to the critical point. Upon departure from the critical point, corrections to the asymptotic power laws appear. The first correction, also known as the Wegner correction [22] contains a new universal scaling function, $g_{1}^{ \pm}(z)$, and a new universal critical exponent $\theta$ (known as the "Wegner exponent"). In the firstorder $\epsilon$-expansion $\theta=\epsilon / 2$ [22].

The Wegner correction arises from the difference between the renormalization-group fixed-point coupling constant $u^{*}$ and the system dependent mean-field value of the coupling constant $u$. When the Wegner correction is included, the Ising field-dependent potential [equation (2)] reads [23]

$$
h_{3}=\left|h_{2}\right|^{2-\alpha} f^{ \pm}(z) 1+\left|h_{2}\right|^{\theta} f_{1}^{ \pm}(z)+\ldots
$$

where $z=h_{1} /\left|h_{2}\right|^{2-\alpha-\beta}$ and $f_{1}^{ \pm} \propto\left(u^{*}-u\right)$. The nonasymptotic (“confluent”) scaling function $f_{1}^{ \pm}$contains additional system-dependent parameters, $\left(u^{*}-u\right)$ and the Ginzburg number, $N_{\mathrm{G}}=u^{2}\left[\rho_{\mathrm{c}}\left(\xi_{0}^{+}\right)^{3}\right]^{-2}$. 
Thus, the scaling power laws are to be complemented by confluent singularities. In terms of physical variables in zero ordering field

$$
\Delta \widetilde{P}=|\Delta \hat{T}|^{2-\alpha}\left[1+\left(\frac{|\Delta \hat{T}|}{t_{\times}}\right)^{\theta}+\left(\frac{|\Delta \hat{T}|}{t_{\times}}\right)^{2 \theta}+\ldots\right],
$$

where the crossover scale $t_{\times} \propto\left(N_{\mathrm{G}}\right)\left(u^{*}-u\right)^{-2}$, which can be considered as the effective Ginzburg number, defines the size of the asymptotic critical region, $|\Delta \hat{T}| \ll t_{\times}$. The amplitude $B_{\mathrm{cr}}$ in the analytic fluctuation-induced contribution to $\phi_{2}$ given by equation (4) also depends on the crossover scale $t_{\times}[17,24]$.

\section{Nonasymptotic asymmetry corrections}

The canonical mapping of the liquid-vapor critical point onto Ising criticality is given by the latticegas model [25]. This model can easily be extended to binary fluids and fluid mixtures through a reassignment of variables and the principle of isomorphism [19]. For the remainder of the text, the liquid-vapor one-component system is only discussed. The lattice-gas model preserves the exact symmetry of uniaxial Ising-type ferromagnets and consequently, the liquid-vapor coexistence curve of the lattice gas is symmetric with respect to the density $\rho$. The order parameter of the lattice gas is the reduced density, $\Delta \hat{\rho}=\left(\rho-\rho_{\mathrm{c}}\right) / \rho_{\mathrm{c}}$. If the liquid and vapor branches of the coexistence curve are denoted by "+" and "-” respectively, the asymmetric portion of the density is given by the excess density

$$
\Delta \hat{\rho}_{\mathrm{d}}=\frac{\Delta \hat{\rho}^{+}+\Delta \hat{\rho}^{-}}{2}
$$

For the lattice gas, $\Delta \hat{\rho}_{\mathrm{d}}=0$. However, real fluids do not possess the symmetry of the Ising model, and in general $\Delta \hat{\rho}_{\mathrm{d}} \neq 0$. Even the coexistence curve of ${ }^{3} \mathrm{He}$, the most symmetric fluid known, exhibits some small asymmetry [26]. In asymmetric systems, the leading behavior is still determined by the Ising-type behavior, and asymmetric corrections appear as sub-leading terms in the quantities like density. In meanfield models of the liquid-vapor critical point, such as the van der Waals model, the asymmetry of the coexistence curve is described by the "law" of rectilinear diameter [27, 28]

$$
\Delta \hat{\rho}_{\mathrm{d}}=D_{1}|\Delta \hat{T}|
$$

where the reduced temperature is defined by $\Delta \hat{T}=\left(T-T_{\mathrm{c}}\right) / T_{\mathrm{c}}$, with $T_{\mathrm{c}}$ being the critical temperature. While some one-component fluids such as xenon [29] seem to asymptotically follow this "law", others, like $\mathrm{SF}_{6}[30]$, show strong deviations from rectilinearity in the critical region.

Models such as the Widom-Rowlinson penetrable-sphere model [31] and Mermin's decorated-lattice models [32, 33] predict non-classical, i.e., non-mean-field, behavior of the excess density. On the basis of these models, a non-classical theory of fluid criticality, known as "revised scaling" [34] was proposed. The formulation of a revised scaling postulates that the Ising scaling fields are analytic functions of the chemical potential $\mu$ and temperature $T$, whereas the lattice gas model assumes that $\mu$ and $T$ are the correct scaling fields. This field mixing produces the following asymptotic behavior:

$$
\Delta \hat{\rho}_{\mathrm{d}} \approx D_{1-\alpha}|\Delta \hat{T}|^{1-\alpha}+D_{1}|\Delta \hat{T}|
$$

Additional theoretical support for a revised scaling came from Nicoll and Zia [35], and Nicoll [36], who performed a field-theoretic (FT) analysis of an asymmetric Landau-Ginzburg-Wilson (LGW) Hamiltonian and found that a revised scaling arises naturally from the inclusion of asymmetric operators in the Hamiltonian. In addition, they found that these asymmetric operators also lead to a non-analytic correction to the excess density characterized by a new asymmetric correction-to-scaling exponent $\theta_{5}$. The excess density predicted by their analysis goes as

$$
\Delta \hat{\rho}_{\mathrm{d}} \approx D_{1-\alpha}|\Delta \hat{T}|^{1-\alpha}+D_{1}|\Delta \hat{T}|+D_{\beta+\theta_{5}}|\Delta \hat{T}|^{\beta+\theta_{5}} .
$$


The universal exponent $\theta_{5}$ was found to be $\theta_{5}=1 / 2+\epsilon=3 / 2$ in the first-order $\epsilon$-expansion, where $\epsilon=4-d$ and $d$ is the spatial dimensionality [37-39]. Working to order $\epsilon^{3}$, Zhang and Zia [40], found their results to be consistent with the bound $\theta_{5} \gtrsim 1.0$.

More recently, Fisher and co-workers [41, 42] have argued for an extended formulation of scaling, originally discussed by Rehr and Mermin [34], which is now known as "complete scaling”. This theory of asymmetric fluid criticality is an extension of the field-mixing in a revised scaling and incorporates the hypothesis of Griffiths and Wheeler [43] that preferable thermodynamic variables do not exist. This concept implies that pressure $P$, chemical potential $\mu$, and temperature $T$ should all be treated on equal footing in any formulation of scaling for the liquid-vapor critical point. The Ising scaling fields should, therefore, be treated as analytic functions of all three. By contrast, a revised scaling assigns a special role to the pressure $P(\mu, T)$ as the field-dependent thermodynamic potential. A complete scaling predicts that the excess density is asymptotically given by

$$
\Delta \hat{\rho}_{\mathrm{d}} \approx D_{2 \beta}|\Delta \hat{T}|^{2 \beta}+D_{1-\alpha}|\Delta \hat{T}|^{1-\alpha}+D_{1}|\Delta \hat{T}|
$$

where $2 \beta \simeq 0.65$. This result clearly differs from the FT prediction, equation (31). In the mean-field approximation, the connection between a complete scaling and the asymmetric Landau expansion has been investigated by Anisimov and Wang [44, 45], who demonstrated that the two approaches appear to be consistent. A complete scaling has also been extended to inhomogeneous fluids by Bertrand and Anisimov [46]. That the penetrable-sphere model does not exhibit complete scaling, has been investigated by Ren et al. [47], who found that this is due to a special symmetry of the model.

In addition to the leading $2 \beta$ term in the excess density, a complete scaling also predicts a divergence in the second derivative of the chemical potential along the coexistence curve

$$
\left(\frac{\mathrm{d}^{2} \mu}{\mathrm{d} T^{2}}\right)_{\mathrm{cxc}} \sim|\Delta \hat{T}|^{-\alpha},
$$

where the subscript cxc denotes the conditions of phase coexistence. The so-called Yang-Yang anomaly derives its name from the Yang-Yang relation [48]

$$
\frac{\rho C_{V}}{T}=\left(\frac{\mathrm{d}^{2} P}{\mathrm{~d} T^{2}}\right)_{\mathrm{cxc}}-\rho\left(\frac{\mathrm{d}^{2} \mu}{\mathrm{d} T^{2}}\right)_{\mathrm{cxc}},
$$

where $C_{V}$ is the isochoric heat capacity. A complete scaling implies that the divergence of the isochoric heat capacity is shared between the second derivatives of the pressure and the chemical potential. By contrast, a revised scaling predicts that $\left(\mathrm{d}^{2} \mu / \mathrm{d} T^{2}\right)_{\mathrm{cxc}}$ remains finite at the critical point. Nicoll's analysis also predicts a non-analytic behavior of the chemical potential, specifically,

$$
\left(\frac{\mathrm{d}^{2} \mu}{\mathrm{d} T^{2}}\right)_{\mathrm{cxc}} \sim|\Delta \hat{T}|^{-\alpha-\beta+\theta_{5}},
$$

however, the relatively large value of $\theta_{5}$ ensures that this quantity remains finite at the critical point.

Fisher and co-workers have found support for a complete scaling in heat capacity measurements [49] and computer simulations of highly asymmetric fluid models [50-54]. Anisimov and Wang have demonstrated that a complete scaling is also supported by the data on liquid-vapor coexistence in highly asymmetric fluids [44, 45]. There is also at least one model that exhibits the type of field mixing characteristic of complete scaling [55, 56]. A complete scaling remains, however, an essentially phenomenological theory.

\section{Complete scaling}

For the liquid-vapor transition, the principle of complete scaling asserts that the scaling fields can be expanded in $\Delta \hat{\mu}, \Delta \hat{T}$, and $\Delta \hat{P}$. In the lowest order approximation, the scaling fields are given by

$$
\begin{aligned}
& h_{1} \simeq a_{1} \Delta \hat{\mu}+a_{2} \Delta \hat{T}+a_{3} \Delta \hat{P}, \\
& h_{2} \simeq b_{1} \Delta \hat{T}+b_{2} \Delta \hat{\mu}+b_{3} \Delta \hat{P}, \\
& h_{3} \simeq c_{1} \Delta \hat{P}+c_{2} \Delta \hat{\mu}+c_{3} \Delta \hat{T}+c_{23} \Delta \hat{\mu} \Delta \hat{T},
\end{aligned}
$$


where the constant coefficients are called mixing coefficients. In general, the complete scaling transformations should include terms of all orders in $\Delta \hat{T}, \Delta \hat{\mu}$, and $\Delta \hat{P}$. Here, we only consider contributions to the excess density $\Delta \hat{\rho}_{\mathrm{d}}$ which are of the order of $|\Delta \hat{T}|$ or lower. Two second-order terms satisfy this criterion, $\Delta \hat{\mu} \Delta \hat{T}$ when added to $h_{3}$ and $\Delta \hat{T}^{2}$ when added to $h_{1}$ and $h_{3}$. However, we have omitted explicit $\Delta \hat{T}^{2}$ terms from the relations for $h_{1}$ and $h_{3}$ since these can be absorbed into the regular, i.e., non-critical, portion of the thermodynamic potential without affecting our results. The exact connection between the transformations, equations (36)-(38), and the excess density will be derived in the following paragraphs. Once this connection is established, one can verify that the remaining second-order terms $\Delta \hat{\mu} \Delta \hat{P}, \Delta \hat{P} \Delta \hat{T}$, $\Delta \hat{P}^{2}$, and $\Delta \hat{\mu}^{2}$ do not need to be included in this approximation.

As discussed by Wang and Anisimov [45] and Bertrand [57], the transformations, equations [36-(38), can be much simplified by selecting normalizations for the scaling fields, adopting a particular value of $\hat{s}_{\mathrm{c}}=s_{\mathrm{c}} / \rho_{\mathrm{c}} k_{\mathrm{B}}$, which is arbitrary in classical thermodynamics, and neglecting higher order terms. Specifically, we choose $\hat{s}_{\mathrm{C}}=(\mathrm{d} P / \mathrm{d} T)_{h_{1}=0, \mathrm{c}}$. These simplifications can be implemented by adopting the following choice of coefficients

$$
\begin{array}{lll}
a_{1}=(1-a), & a_{2}=-a \hat{s}_{\mathrm{c}}, & a_{3}=a, \\
b_{1}=1, & b_{3}=0, \\
c_{1}=1, & b_{2}=b, & c_{3}=-\hat{s}_{\mathrm{c}}, \quad c_{23}=c .
\end{array}
$$

When these coefficients are substituted into the complete scaling transformations, we find that the transformations reduce to

$$
\begin{aligned}
& h_{1}=\Delta \hat{\mu}+a \Delta \widetilde{P}, \\
& h_{2}=\Delta \hat{T}+b \Delta \hat{\mu}, \\
& h_{3}=\Delta \widetilde{P}+c \Delta \hat{\mu} \Delta \hat{T},
\end{aligned}
$$

where $\Delta \widetilde{P}$ is defined by equation (21). In the mean-field approximation, $\Delta \hat{\mu} \sim|\Delta \hat{T}|^{3 / 2}$ and $\Delta \hat{P} \sim|\Delta \hat{T}|^{2}$, so that each asymmetric term in the complete scaling transformations is smaller than the leading term by a factor of $|\Delta \hat{T}|^{1 / 2}$. The revised scaling transformations are reproduced in the absence of pressure mixing $(a=0)$, and the lattice gas model is recovered when all mixing coefficients are set to zero $(a=b=c=0)$.

The physical densities can be found in terms of the scaling densities from equations (42)-(44) with the result

$$
\begin{aligned}
& \Delta \hat{\rho}=\frac{\phi_{1}+b \phi_{2}-c \Delta \hat{T}}{1-a \phi_{1}}, \\
& \Delta \hat{s}=\frac{\phi_{2}}{1-a \phi_{1}},
\end{aligned}
$$

where $\phi_{1}$ and $\phi_{2}$ are given by equations (3) and (4), respectively. To the leading order in the asymmetry and reduced temperature, these expressions are given by

$$
\begin{aligned}
& \Delta \hat{\rho} \simeq \phi_{1}+a\left(\phi_{1}\right)^{2}+b \phi_{2}-c \Delta \hat{T}, \\
& \Delta \hat{s} \simeq \phi_{2} .
\end{aligned}
$$

When the scaling densities presented in equations (3) and (4) are substituted into equation (47), the complete scaling excess density introduced in equation (32) is reproduced with the coefficients

$$
\begin{aligned}
D_{2 \beta} & =a\left(B_{0}\right)^{2}, \\
D_{1-\alpha} & =-b \frac{A_{0}^{-}}{1-\alpha}, \\
D_{1} & =B_{\mathrm{cr}}+c .
\end{aligned}
$$

We note that the leading $2 \beta$ term is proportional to the pressure mixing coefficient $a$. The same is true of the Yang-Yang anomaly, which follows from the first complete scaling relationship, equation (42), as

$$
\left(\frac{\mathrm{d}^{2} \hat{\mu}}{\mathrm{d} \hat{T}^{2}}\right)_{\mathrm{cxc}} \simeq-a\left(\frac{\mathrm{d}^{2} \hat{P}}{\mathrm{~d} \hat{T}^{2}}\right)_{\mathrm{cxc}}=-a A_{0}^{-}|\Delta \hat{T}|^{-\alpha},
$$


where, to the leading order, the coexistence curve is defined by $h_{1}=0$.

Complete scaling also predicts the effects of fluid asymmetry on other thermodynamic properties. In particular, the physical susceptibilities, such as the isothermal compressibility, volumetric expansivity, and the heat capacity are found to be combinations of all three scaling susceptibilities: "strong” $\chi_{1}$, "weak" $\chi_{2}$, and "cross" $\chi_{12}[45]$.

\section{Discussion and conclusion}

As shown in previous section, asymmetric fluid criticallity generally introduces three additional, nonuniversal and independent, amplitudes associated with the mixing of physical fields into the Ising scaling fields. However, as the comparison between a complete scaling and the FT approach to asymmetric fluid criticality shows [58], the complete scaling and FT equations of state are nearly identical, except that the FT equation of state has an additional term responsible for the asymmetric correction-to-scaling exponent $\theta_{5}$.

As a result, the asymtery-induced excess density can be written

$$
\Delta \hat{\rho}_{\mathrm{d}} \approx D_{1-\alpha}|\Delta \hat{T}|^{1-\alpha}+D_{2 \beta}|\Delta \hat{T}|^{2 \beta}+D_{1}|\Delta \hat{T}|+D_{\beta+\theta_{5}}|\Delta \hat{T}|^{\beta+\theta_{5}},
$$

where $D_{\beta+\theta_{5}} \propto u_{5}^{\text {eff }}$, a new nonasymptotic amplitude. For many practical applications, the contribution from $\theta_{5}$ can be neglected. In this regime, the complete scaling and FT approaches are equivalent. In practice, the number of independent amplitudes may be constrained by a particular equation of state.

There is an analogy between the asymmetric correction-to-scaling exponent $\theta_{5}$ and the Wegner correction-to-scaling exponent $\phi \simeq \epsilon / 2$ [22]. The Wegner correction arises from the difference between the renormalization-group fixed-point coupling constant $u^{*}$ and the system-dependent mean-field value of the coupling constant $u$ [cf. equation [26)]. As in the case of the Wegner correction, which is associated with an additional critical amplitude $u^{*}-u$, the $\theta_{5}$ exponent is associated with the new critical amplitude $u_{5}^{\text {eff }}$ which is the difference between the fifth-order coefficient in the asymmetric Landau expansion and the amplitude of the asymmetry of the gradient term in the effective Hamiltonian [58]. If $u_{5}^{\text {eff }}=0$, $h_{3}$ includes only the leading asymmetric terms. In this particular case, complete scaling becomes exact. After the complete scaling has taken care of the leading asymmetric corrections by the mixing of physical fields into the scaling field, the field dependent potential could be extended as

$$
h_{3} \approx\left|h_{2}\right|^{2-\alpha} f^{ \pm}(z)\left[1+\left|h_{2}\right|^{\theta} f_{1}^{ \pm}(z)+\left|h_{2}\right|^{\theta_{5}} f_{\mathrm{asym}}^{ \pm}(z)\right],
$$

where $f_{\text {asym }}^{ \pm} \propto u_{5}^{\text {eff }}$. However, there is a significant difference between these two corrections-to-scaling. Unlike $\theta_{5}$, the exponent $\theta$ vanishes in the mean-field approximation $\epsilon=0$. This explains why the Wegner correction can be consistently omitted in the mean-field approximation. The same is not true of $\theta_{5}$, because in the mean-field approximation $\theta_{5}=1 / 2$.

\section{Acknowledgements}

I thank C.E. Bertrand, J.F. Nicoll, and J.V. Sengers for collaboration and M.E. Fisher for discussions and comments. I also appreciate long-term fruitful interactions with scientists from the Institute for Condensed Matter Physics, the National Academy of Sciences of Ukraine, in particular with I.R. Yukhnovskii and M.P. Kozlovskii who made important contributions to the physics of critical phenomena and phase transitions [59, 60]. 


\section{References}

1. Fisher M.E., In: Critical Phenomena, Hahne F.J.W. (Ed.), Lecture Notes in Physics Vol. 186, Springer, Berlin, 1982.

2. Domb C., The Critical Point: A Historical Introduction to the Modern Theory of Critical Phenomena, Taylor and Francis, London, 1996.

3. Anisimov M.A., Critical Phenomena in Liquids and Liquid Crystals, Gordon and Breach, Philadelphia, 1991.

4. Sengers J.V., Shanks J.G., J. Stat. Phys., 2009, 137, 857; doi 10.1007/s10955-009-9840-z

5. Landau L.D., Lifshitz E.M., Statistical Physics, Pergamon, New York, 1958.

6. Sengers J.V., Sengers J.M.H.L., Ann. Rev. Phys. Chem., 1986, 37, 189; doi 10.1146/annurev.pc.37.100186.001201

7. Liu A.J., Fisher M.E., Physica A, 1989, 156, 35; doi 10.1016/0378-4371(89)90109-X

8. Guida R., Zinn-Justin J., J. Phys. A: Math. Gen., 1998, 31, 8103; doi 10.1088/0305-4470/31/40/006

9. Campostrini M., Pelissetto A., Rossi P., Vicari E., Phys. Rev. E, 1999, 60, 3526; doi 10.1103/PhysRevE.60.3526.

10. Campostrini M., Pelissetto A., Rossi P., Vicari E., Phys. Rev. E, 2002, 65, 066127; doi 10.1103/PhysRevE.65.066127

11. Pelissetto A., Vicari E., Phys. Rep., 2002, 368, 549; doi 10.1016/S0370-1573(02)00219-3

12. Fisher M.E., Zinn S.-Y., J. Phys. A: Math. Gen., 31, L629 (1998); doi 10.1088/0305-4470/31/37/002

13. Haupt A., Straub J., Phys. Rev. E, 1999, 59, 1795; doi 10.1103/PhysRevE.59.1795

14. Anisimov M.A., Sengers J.V., In: Equations of State for Fluids and Fluid Mixtures, Sengers J.V., Kayser R.F., Peters C.J., White H.J. (Jr.), (Eds.), Elsevier, Amsterdam, 2000, p. 381.

15. Anisimov M.A., Thoen J., In: Heat Capacities of Liquids and Vapours, Wilhelm E., Trevor T.M. (Eds.), Royal Society of Chemistry, Cambridge, 2010, Chapter 14, p. 307.

16. Behnejad H., Sengers J.V., Anisimov M.A., In: Applied Thermodynamics of Fluids, Goodwin A., Peters C., Sengers J.V. (Eds.), Royal Society of Chemistry, Cambridge, 2010, Chapter 10, p. 321.

17. Anisimov M.A., Kiselev S.B., Sengers J.V., Tang S., Physica A, 1992, 188, 487; doi 10.1016/0378-4371(92)90329-O

18. Landau L.D., Lifshitz E.M., Statistical Physics, 3rd Edn., Part 1, Pergamon, Oxford, 1980.

19. Anisimov M.A., Gorodetskii E.E., Kulikov V.D., Sengers J.V., Phys. Rev. E, 1995, 51, 1199; doi 10.1103/PhysRevE.51.1199

20. Schofield P., Phys. Rev. Lett., 1969, 22, 606; doi 10.1103/PhysRevLett.22.606

21. Brézin E., Wallace D.J., Wilson K.G., Phys. Rev. Lett., 1972, 29, 591; doi 10.1103/PhysRevLett.29.591

22. Wegner F.J., Phys. Rev. B, 1972, 5, 4529; doi 10.1103/PhysRevB.5.4529

23. Pelissetto A., Vicari E., Phys. Rep., 2002, 368, 549; doi 10.1016/S0370-1573(02)00219-3

24. Kim Y.C., Anisimov M.A., Sengers J.V., Luijten E., J. Stat. Phys., 2003, 110, 591; doi 10.1023/A:1022199516676

25. Lee T.D., Yang C.N., Phys. Rev., 1952, 87, 410; doi 10.1103/PhysRev.87.410.

26. Hahn I., Weilert M., Zhong F., Barmatz M., J. Low. Temp. Phys., 2004, 137, 579; doi 10.1007/s10909-004-0893-8

27. Cailletet L., Mathias C.R., Hebd C.R., Seances Acad. Sci., 1886, 102, 1202.

28. Cailletet L., Mathias C.R., Hebd C.R., Seances Acad. Sci., 1887, 104, 1563.

29. Närger U., Balzarini D.A., Phys. Rev. B, 1990, 42, 6651; doi 10.1103/PhysRevB.42.6651

30. Weiner J., Langley K.H., Ford N.C. (Jr.), Phys. Rev. Lett., 1974, 32, 879; doi 10.1103/PhysRevLett.32.879.

31. Widom B., Rowlinson J.S., J. Chem. Phys., 1970, 52, 1670; doi 10.1063/1.1673203

32. Mermin N.D., Phys. Rev. Lett., 1971, 26, 169; doi 10.1103/PhysRevLett.26.169

33. Mermin N.D., Phys. Rev. Lett., 1971, 29, 957; doi 10.1103/PhysRevLett.26.957

34. Rehr J.J., Mermin N.D., Phys. Rev. A, 1973, 8, 472; doi 10.1103/PhysRevA.8.472

35. Nicoll J.F., Zia R.K.P., Phys. Rev. B, 1981, 23, 6157; doi 10.1103/PhysRevB.23.6157

36. Nicoll J.F., Phys. Rev. A, 1981, 24, 2203; doi 10.1103/PhysRevA.24.2203

37. Vause C., Sak J., Phys. Rev. A, 1980, 21, 2099; doi 10.1103/PhysRevA.21.2099

38. Vause C., Sak J., Phys. Rev. A, 1980, 23, 1562; doi 10.1103/PhysRevA.23.1562

39. Ley-Koo M., Green M.S., Phys. Rev. A, 1981, 23, 2650; doi 10.1103/PhysRevA.23.2650.

40. Zhang F.C., Zia R.K.P., J. Phys. A: Math. Gen., 1982, 15, 3303; doi 10.1088/0305-4470/15/10/032

41. Fisher M.E., Orkoulas G., Phys. Rev. Lett., 2000, 85, 696; doi 10.1103/PhysRevLett.85.696

42. Kim Y.C., Fisher M.E., Orkoulas G., Phys. Rev. E, 2003, 67, 061506; doi 10.1103/PhysRevE.67.061506

43. Griffiths R.B., Wheeler J.C., Phys. Rev. A, 1970, 2, 1047; doi 10.1103/PhysRevA.2.1047

44. Anisimov M.A., Wang J., Phys. Rev. Lett., 2006, 97, 025703; doi 10.1103/PhysRevLett.97.025703

45. Wang J., Anisimov M.A., Phys. Rev. E, 2007, 75, 051107; doi 10.1103/PhysRevE.75.051107.

46. Bertrand C.E., Anisimov M.A., Phys. Rev. Lett., 2010, 104, 205702; doi 10.1103/PhysRevLett.104.205702

47. Ren R., O’Keeffe C.J., Orkoulas G., J. Chem. Phys., 2006, 125, 144505; doi 10.1063/1.2356862

48. Yang C.N., Yang C.P., Phys. Rev. Lett., 1964, 13, 303; doi 10.1103/PhysRevLett.13.303

49. Orkoulas G., Fisher M.E., Üstün C., J. Chem. Phys., 2000, 113, 7530; doi 10.1063/1.1308284

50. Orkoulas G., Fisher M.E., Panagiotopoulos A.Z., Phys. Rev. E, 2001, 63, 051507; doi 10.1103/PhysRevE.63.051507

51. Kim Y.C., Fisher M.E., Chem. Phys. Lett., 2005, 414, 185; doi 10.1016/j.cplett.2005.07.105 
52. Kim Y.C., Fisher M.E., Luijten E., Phys. Rev. Lett., 2003, 91, 065701; doi 10.1103/PhysRevLett.91.065701

53. Kim Y.C., Fisher M.E., Phys. Rev. E, 2003, 68, 041506; doi 10.1103/PhysRevE.68.041506

54. Kim Y.C., Phys. Rev. E, 2005, 71, 051501; doi 10.1103/PhysRevE.71.051501

55. Felderhof B.U., Fisher M.E., Ann. Phys., 1970, 56, 176.

56. Felderhof B.U., Fisher M.E., Ann. Phys., 1970, 56, 217.

57. Bertrand C.E., Asymmetric Fluid Criticality, Ph. D. Thesis, University of Maryland, 2011.

58. Bertrand C.E., Nicoll J.F., Anisimov M.A., Phys. Rev. E, 2012, 85, 031131; doi 10.1103/PhysRevE.85.031131

59. Yukhnovskii I.R., Phase Transitions of the Second Order. Collective Variables Method, World Scientific, Singapore, 1987.

60. Yukhnovskii I., Kozlovskii M., Pylyuk I., Microscopic Theory of Phase Transitions in Three-Dimensional Systems, Evrosvit, Lviv, 2001 (in Ukrainian).

\title{
Універсальність чи неуніверсальність в асиметричній критичності плинів
}

\begin{abstract}
М.А. Анісімов
Інститут фізичної науки і технології, Університет Мариленду, Коледж Парк, MD 20742, США

Критичні явища в реальних плинах демонструють комбінацію універсальних рис, спричинених розбіжністю далекосяжних флуктуацій густини, і неуніверсальних (системо залежних) рис, пов'язаних із специфічними міжмолекулярними взаємодіями. Асимптотично всі плини належать до класу універсальності моделі Ізинга. Асимптотичні степеневі закони для термодинамічних властивостей описуються двома незалежними універсальними критичними показниками і двома незалежними неуніверсальними критичними амплітудами; решту критичних амплітуд можна отримати з універсальних співвідношень. Неуніверсальні критичні параметри (критична температура, тиск і густина) можуть бути включені в одиниці цих властивостей. Неасимптотичну критичну поведінку плинів можна поділити на дві частини, симетричну ("ізингоподібну”) і асиметричну (“плиноподібну”). Симетрична неасимптотична поведінка містить новий універсальний показник (показник Вегнера) і системо залежний масштаб кросоверу (число Гінзбурга), пов'язаний з областю дії міжмолекулярних взаємодій, тоді як асиметричні риси взагальному описуються додатковим універсальним показником і трьома неасимптотичними амплітудами, пов'язаними зі змішуванням фізичних полів у скейлінгових полях.
\end{abstract}

Ключові слова: плини, критична точка, універсальність, повний скейлінг 